\title{
Efficacy of some antibiotics and some metal complexes (Nano-formula) that could increase their effectiveness during COVID-19
}

\author{
Reham Z. Hamza *, Zain A. Sheshah, Reham H. Suleman, Norah F. Al-Juaid, Nejoud A. Hamed and Maram A. Al- \\ Juaid
}

Biology Department, College of Sciences, Taif University, Taif 11099, Saudi Arabia.

International Journal of Biological and Pharmaceutical Sciences Archive, 2022, 03(01), 008-014

Publication history: Received on 01 December 2021; revised on 08 January 2022; accepted on 10 January 2022

Article DOI: https://doi.org/10.53771/ijbpsa.2022.3.1.0021

\begin{abstract}
Medicinal applications of metals and their complexes are of elevating clinical importance especially during COVID-19 pandemic. Antibiotics like (Ceftriaxone "CFx"- Erythromycin"Ery") chemically complexes with different metals by using methanol and continuous stirring for $3 \mathrm{~h}$ and then the colored precipitates were obtained and then dried for further chemical analysis and confirming the chemical structure by TEM analysis and then measuring total antioxidant capacity of the complex and performing DPPH assay for the antibiotic drug and its different metal complexes like Se (II) , Mg(II) and $\mathrm{Zn}$ (II). complexes were studied and characterized based on transmission electron microscopy (TEM). Chemical data revealed that CFx and Ery have great ability to complexes with these metals and give different chemical structure. Antioxidant capacities, anti-inflammatory activities were carried out in vitro. The metal complexed with antibiotics "combination" exhibited potent antioxidant and ant inflammatory activities and suppressed oxidative stress that could adjust the pulmonary disturbances and alterations induced by pneumenia. In conclusion, Antibiotics/metal drug complexes could produce a high synergistic effects against oxidative stress and viral infection symptoms in some cases and can be considered potential ameliorative therapy against pulmonary dysfunction, which may benefit against COVID19 pandemic.
\end{abstract}

Keywords: Antibiotics; Ceftriaxone; Erythromycin; Magnesium; Selenium; Zinc; Anti-diabetic agents; Pulmonary functions; Oxidative damage

\section{Introduction}

Ceftriaxone antibiotic belongs to the $3^{\text {rd }}$ generation of cephalosporin antibiotic drugs. It exhibits a broad spectrum of activity against two types of bacteria either Gram-negative or positive pathogens and infections. It's broad spectrum includes lower respiratory infections and urinary tract infections, inflammatory diseases as well as surgical infections. The drug is widely used due to its broad spectrum of antibacterial activity and very rare side effects [1].

Some studies manufactured a multi-linked compound photocatalyst that combines the advantages of homogeneous and heterogeneous bonds. This multi-linking structure facilitated the separation of electrons and holes through the multistep energy level structure. Some previous studies have examined the catalytic performance for the simultaneous removal of ceftriaxone sodium and chromium (VI) [2]. They observed that the degradation rate of ceftriaxone sodium decreased significantly while the rate of decrease of $\mathrm{Cr}$ (VI) increased further (by 7.4 times). When ceftriaxone sodium and $\mathrm{Cr}$ (VI) were treated simultaneously, the electrons Cr (VI) decreased, resulting in reduced 02 generation. The simultaneous removal of ceftriaxone sodium and chromium (VI) effectively increased the use of photosynthetic electron holes [2].

\footnotetext{
* Corresponding author: Reham Z Hamza

Biology Department, College of Sciences, Taif University, Taif 11099, Saudi Arabia.

Copyright $(2022$ Author(s) retain the copyright of this article. This article is published under the terms of the Creative Commons Attribution Liscense 4.0.
} 
Given the prevalence of vitamin and mineral supplement use among consumers and the potential for vitamin- and mineral-drug interactions, as well as oral and systemic adverse effects of excess consumption, oral health care providers (OHCPs) should ask all patients about their use. The challenges for OHCPs are how to recognize oral and systemic manifestations of these interactions and how to safely manage the care of these patients while avoiding potential interactions [3].

Topical antibiotics are the main step in the treatment of mild to moderate acne vulgaris. Erythromycin is one of the effective topical therapies for this disease. Zinc sulfate $5 \%$ solution was reported to be effective in treatment of acne vulgaris and rosacea and Zinc is considered a wide broad spectrum antibacterial element [4]

Erythromycin, one of the most widely antibiotics, has been detected in a lot of aquatic environments. So, Ery ecotoxicity should deserve more and high economic attention. In previous study, blue mussels were exposed to Ery to explore its potential physiological toxicity. After 2 days acute and 7 days sub-acute exposure to Ery, glutathione S-transferase (GST) and catalase (CAT) activities were determined and metabolic responses were analyzed by $\left({ }^{1} \mathrm{H}-\mathrm{NMR}\right)$. The results revealed that GST was approximately 1.6 times higher in mussels exposed to about $200 \mathrm{mg} / \mathrm{L}$ and higher concentrations. CAT was 1.9 times higher in mussels exposed to $200 \mathrm{mg} / \mathrm{L}$, indicating that Ery exposure led that blue mussels enhanced antioxidant responses [5].

The toxic effects of "Ery" and enrofloxacin (ENR), applied individually or in combination, on Chlorella vulgaris were investigated in this study, as well as the toxic mechanisms. Antioxidant protection system and photosynthesis were implicated in toxic processes, with findings showing that antibiotic treatments increased antioxidant enzyme activity as well as malondialdehyde (MDA) and glutathione (GSH) levels. Furthermore, the rise was greater in the joint exposure treatment, suggesting that the antioxidant protection mechanism was affected synergistically [5].

The aim of this study was to see how Labeo rohita reacted to different concentrations of erythromycin $(10,50$, and 100 $\mathrm{g} / \mathrm{L}$ ) in the short (96 hours) and long-term (35 days) antioxidant responses. We focused on two studies (short and longterm), and found that the erythromycin-treated fingerlings had higher glutathione peroxidase (GPx) and lipid peroxidation (LPO) activities in the gills and liver than the control groups in both the short and long-term study periods [6].

Additionally, erythromycin induces oxidative stress in aquatic species (L. rohita), and this data could serve as a useful baseline for molecular toxicology to track antibiotics' effects on non-target organisms.

\subsection{Bacterial co-infection with covid-19}

Bacterial co-pathogens are often present in viral respiratory infections, and they are a significant cause of morbidity and mortality. In patients infected with SARS-CoV-2, the prevalence of bacterial infection is uncertain[6].

The percentage of SARS-CoV-2 patients who are administered antibiotics is unknown, and this could lead to patient damage and global antibiotic resistance [7].

The aim of this study was to determine the prevalence of antibiotic prescribing in COVID-19 patients, as well as the factors that influence antibiotic prescribing.

Coronavirus disease-19 (COVID-19) is characterized by a wide variety of symptoms, from asymptomatic to serious illness and death. The study's aim was to thoroughly explain the clinical features and outcomes in critically ill COVID19 patients in Saudi Arabia [8].

Here, in this article, the $\mathrm{Zn}(\mathrm{II}), \mathrm{CU}(\mathrm{II}), \mathrm{Mg}(\mathrm{II})$ and Se(II) and other metals ceftriaxone complexes were synthesized and characterization using SEM and TEM analyses techniques. In addition, the antioxidant activity of complexes (DPPH) assay was investigated.

\section{Material and methods}

\subsection{Chemicals and Analyses}

All chemicals (Ceftriaxone, Erythromycin , zinc(II) chloride, Magnesium chloride and selenium chloride) and other used metals were high analytical chemical grade and used without purification. metals complex with Antibiotics were synthesized by the following method. 


\subsection{Synthesis of Cfx or Ery/metals complexes}

$1 \mathrm{mmol}$ of either "CFx or "Ery" dissolved in (30 mL) methanol and $1 \mathrm{mmol}$ of each salts was also dissolved in methanol and then mixed, Then, the product was stirred for $4 \mathrm{~h}$ at room temperature. After stirring, the resulting yellow and then green products were filtered, evaporated slowly at room temperature overnight, then washed with few amounts of methanol, Chemical compounds as shown in Fig.1.

\subsection{TEM analysis}

\begin{tabular}{|l|l|}
\hline Type of analysis & Models \\
\hline TEM & JEOL 100s microscopy \\
\hline SEM & Quanta FEG 250 equipment \\
\hline
\end{tabular}

\subsection{Total antioxidant capacity}

Antioxidant capacity of both metal complexes were evaluated by ABTS $\bullet+$ radical cation decolourisation assay. The absorbance of the ABTS $\bullet+$ solution was equilibrated to $0.70( \pm 0.02)$ by dilution at room temperature, then 1 ml was mixed with $10 \mu \mathrm{l}$ of each of the complex and their absorbance was measured at $734 \mathrm{~nm}$ after 6 min [9] (Fig.1).

\subsection{DPPH radical scavenging assay}

The study for the antioxidant capacity of the metal complexes were compared by the DPPH scavenging assay according to Mahakunakorn et al. [10]. Different concentrations $(0-100 \mu \mathrm{g} / \mathrm{ml})$ of the complexes were mixed with equal volume of ethanol. Then $50 \mu \mathrm{l}$ of DPPH solution ( $1 \mathrm{mM}$ ) was pipetted into the solution and stirred. The optical density (OD) of the resulting solution was immediately measured at $517 \mathrm{~nm}$.

\subsection{Statistical analysis}

Statistical analysis was done using SPSS software version 27 (IBM, 2020) and Open Epi version 2.3.1 [11]. Graphical and tabular presentation was done. Data were summarized as mean and standard deviation. One way ANOVA and post hoc power were used to analyze data. A P-value of $<0.05$ was accepted as statistically significant [12].

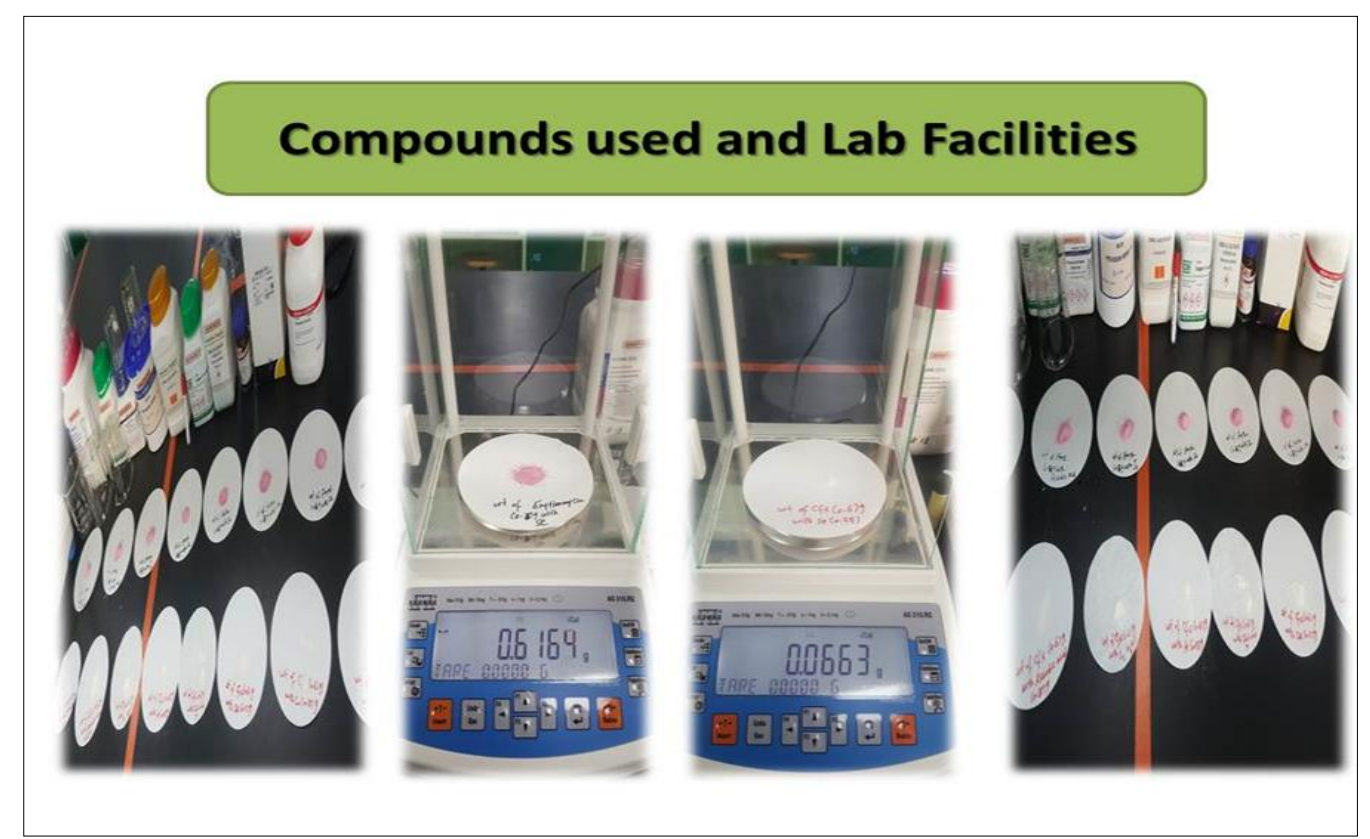




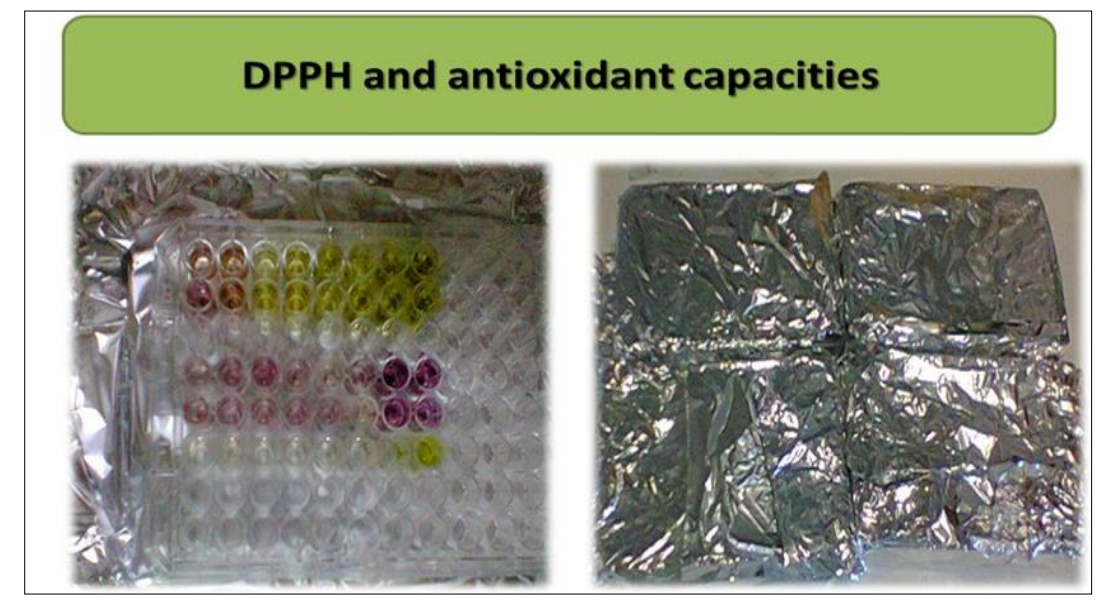

Figure 1 Chemical compounds and summarization of practical work

\section{Results}

\subsection{TEM characterization of some CFx or Ery /metal complexes}

Figures (2 and 3) showed Scanning electron microscopy (SEM) and the transmission electron microscopy (TEM) showing homogenous clear from. photograph of the synthesized some metals with Antibiotics (CFx and Ert) complexes. The uniform matrix of the synthesized complex was clear. Black, spherical dots with nanorange were observed in the synthesized complex.
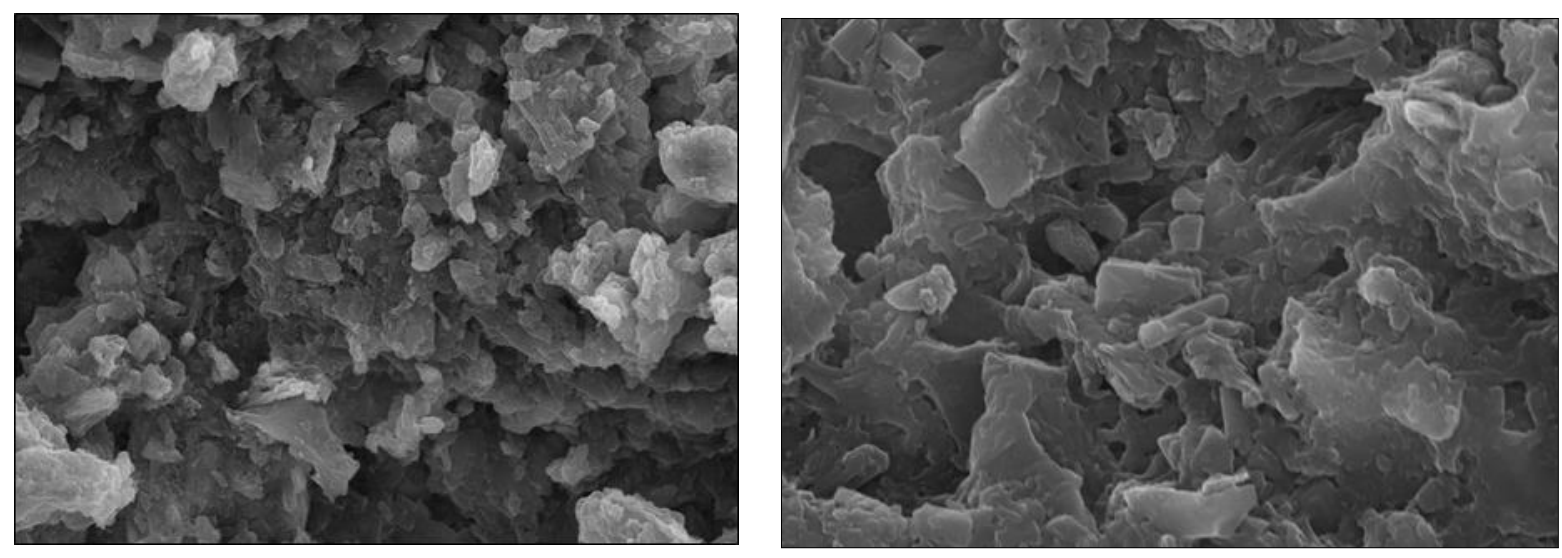

Figure 2 SEM of CFx or Ery metal complexes

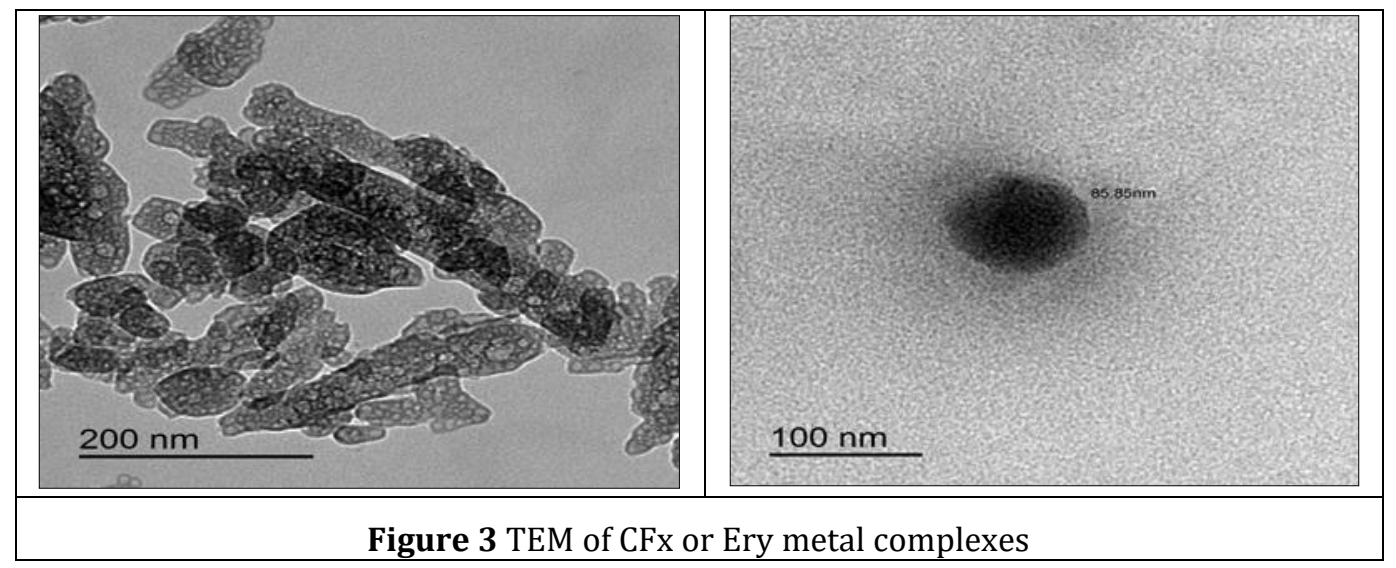




\subsection{Antioxidant capacities of the metal complexes}

Total antioxidant activity The percentage inhibition of absorbance was calculated as a function of the concentration of standard and sample to determine the equivalent antioxidant concentration (TEAC). The TEAC value of CFx/metal complexes were found to be $0.273 \pm 0.013$

Table 1 Total antioxidant capacities of the CFX/metal complexes

\begin{tabular}{|c|c|}
\hline Name of Complex & Total equivalent antioxidant capacity \\
\hline $\mathrm{CFx} / \mathrm{Se}$ & $0.478 \pm 0.01^{\mathrm{c}}$ \\
\hline $\mathrm{CFx} / \mathrm{Zn}$ & $0.397 \pm 0.03^{\mathrm{b}}$ \\
\hline $\mathrm{CFx} / \mathrm{Mg}$ & $0.361 \pm 0.04 \mathrm{a}$ \\
\hline
\end{tabular}

\section{Discussion}

Obtaining a cure or treatment for symptoms for COVID-19 is the most important worldwide concern in 2020.0ne of the treatment protocols for COVID-19 uses Azomycthin or Eryhromycin alone or in conjugation with other compounds and also with clinical major insights to CFx. Providing additional insight into the chemistry of these antibiotics by examining its interactions that may help researchers to improve the treatment protocols for COVID-19. This work investigated the formation of some metal antibiotics complexes arising from the complexation of the used antibiotics in methanol solvent. The structures and morphologies of the synthesized complexes were confirmed partially by SEM and TEM.

Some antibiotics as (Cfx-Na) has broad spectrum activity against both Gram-negative and Gram-positive bacteria. By inhibiting bacterial cell synthesis, often used to treat community-acquired pneumonia [1].

The antibiotic drugs used as donors as moxifloxacin and ceftriaxone and they are highly potent against many types of bacteria and, therefore, widely used worldwide [13-15].

An important member of this family is moxifloxacin. MOX is a fourth-generation antibiotic with a broad spectrum of antimicrobial activity against Gram--ve and +ve o, respiratory tract pathogens and anaerobic bacteria [14].

Ceftriaxone is a $3^{\text {rd }}$-generation cephalosporin antibiotic with a broad spectrum of activity against a variety of infections caused by both Gram-ve and +ve pathogens. This antibiotic has several remarkable properties such as few and rare side effects, and a broad spectrum of antibacterial efficacy; therefore, it has a long list of clinical applications including intra-abdominal infections, and infections of the skin and skin structure, urinary tract infections, and lower respiratory infections [15].

The metals play an important role in case of complexation with antibiotics. Antibiotics in combination with calcium salts causes kidney stones because they become insoluble deposits. This gave me a start in his ability to coordinate iron, cobalt, and other minerals to study the properties of the link between them and the antibiotic .

Copper has been published in particular as a collection of research papers relating to the composition and physical and chemical studies of several complexes .

Zinc is an important and essential metal involved in a lot of biological processes due to its potent antiviral and antioxidant functions as a cofactor and structural elements [16].

- $\quad$ The most important role of $\mathrm{Zn}$ is its efficacy for the immune system. As, Zn functioning part of leukocytes and lymphocytes mechanism of action (Immune soldiers).

- $\quad$ Zn plays a vital role involved in relieving of inflammatory responses.

- $\quad$ Zn is also a major component of the nutritional immunity

- $\quad$ Alteration of Zn behavioral status significantly affects immune response resulting in elevated the susceptibility to inflammatory and infectious diseases including acquired immune deficiency syndrome, malaria, measles and mainly pneumonia. 
Under Zn deficiency state, organisms are high susceptible to toxin-producing bacteria or viral pathogens that could activate adenylate cyclases and stimulating chloride secretion, causing diarrhea and diminishing absorption of nutrients.

$\mathrm{Zn}$ modifies the antiviral and bacterial immunity and regulates the inflammatory response, and it is antiviral by inhibiting the RNA polymerase for SARS- Cov, it has a preventive and adjunctive treatment for COVID 19, improving the status of zinc may reduce the risk of developing a common bacterial infection by improving the removal of the mucous membrane that it is caused by a ventilator [16].

Magnesium may contribute to the onset, progression, and severity of COVID-19. If it is deficient, there is a decrease in $\mathrm{T}$ cells, an increase in the plasma concentration of inflammatory cytokines, and endothelial dysfunction. Magnesium may be involved in the process of killing bacteria inside cells [17].

\section{Conclusion}

Our findings revealed that There is a desired and urgent need for new antimicrobial compounds to combat the growing threat of widespread bacterial and viral resistance. New classes of antibiotics are urgently required which could by synthesized by complexation with metals. Metal complexes are currently in clinical development for the treatment of malaria , may be potent against COV-SAR 2 and neurodegenerative diseases. However, only little attention has been paid to the application of antibiotic metal complexes as potential antimicrobial compounds. Our results confirmed that the Antibiotics/Se , Mg and $\mathrm{Zn}$ complexes are of highly efficient and safe for treating oxidative injury induced by bacterial and viral storm. These results open new gate to develop therapeutic strategies and prevention of sever diseases associated complications, thereby providing hope for alleviating the symptoms of COVID-19 or other associated disorders.

\section{Recommendation}

The present results indicated that some widely used antibiotics as (Ceftriaxone and Erythromycin) are considered as potent antioxidant and anti-inflammatory agent and the prospective studies indicates the great role of the metals in enhancing the capacities of these compounds to act as potent antiviral agents that could be of great importance for protection especially during COVID-19 pandemic and could be considered as protective agent against the severe symptoms of COVID-19.

\section{Compliance with ethical standards}

\section{Acknowledgments}

Authors (Supervisor staff member and students of graduation project at Biology Department) acknowledge and thankful to Biology Department, College of Sciences, Taif University, Taif, Saudi Arabia, for using Department's laboratories for performing experimental work.

\section{Disclosure of conflict of interest}

The author declares that there are not any conflicts of interest.

\section{References}

[1] Moamen S. Refat, T. Altalhi, Hammad Fetooh, Amnah M. Alsuhaibani, Reham F. Hassan. In neutralized medium five new $\mathrm{Ca}(\mathrm{II}), \mathrm{Zn}(\mathrm{II}), \mathrm{Fe}(\mathrm{III}), \mathrm{Au}(\mathrm{III})$ and $\mathrm{Pd}(\mathrm{II})$ complexity of ceftriaxone antibiotic drug: Synthesis, spectroscopic, morphological and anticancer studies. Journal of Molecular Liquids. 2021; 322: 114816.

[2] Jingjing Xu, Jiawei Zhu, Mindong Chen. Simultaneous removal of ceftriaxone sodium and Cr(VI) by a novel multijunction (p-n junction combined with homojunction) composite photocatalyst: BiOI nanosheets modified cakelike anatase-rutile TiO2. Journal of Molecular Liquids. 2020; 320: 114479.

[3] Mark Donaldson and Riva Touger-Decker JADA. Vitamin and mineral supplements Friend or foe when combined with medications?.the journal of the American Dental Association. 2014; 145(11): 1153-1158. 
[4] Hayder R. Al-Hamamy, Khalifa E. Sharquie, Adil A. Noaimi, Wajeeh N. Hussein. Topical Erythromycin-Zinc acetate complex Lotion versus topical Erythromycin Gel in treatment of mild to moderate Acne vulgaris. Dermatol. 2014; 5(4): 347-351.

[5] Ruoyu Liang, Xiuqing Shao, Yajuan Shi, Longxin Jiang, Guoxiang Han. Antioxidant defenses and metabolic responses of blue mussels (Mytilus edulis) exposed to various concentrations of erythromycin. Journal Pre-proof. 2018.

[6] Bradley J, et al. Antibiotic prescribing in patients with COVID-19: rapid review and meta-analysis. Clinical Microbiology and Infection. 2021; 26(12): 1622-1629.

[7] Langford BJ, So M, Raybardhan S, Leung V, Westwood D, MacFadden DR, Soucy J-PR, Daneman N. Bacterial coinfection and secondary infection in patients with COVID-19: a living rapid review and meta-analysis Clinical Microbiology and Infection. 2020.

[8] Khalid A, et al. Clinical Features and Outcomes of Critically Ill Patients with Coronavirus Disease 2019 (COVID19): A Multicenter Cohort Study. International Journal of Infectious Diseases. 2021.

[9] Mandal S, Hazra B, Sarkar R, Biswas S, Mandal N. Assessment of the antioxidant and reactive oxygen species scavenging activity of methanolic extract of Caesalpinia crista leaf. Evid Based Complement Alternat Med. 2009.

[10] Mahakunakorn P, Tohda M, Murakami Y, Matsumoto K, Watanabe H. Antioxidant and free radical-scavenging activity of Choto-san and its related constituents. Biol Pharm Bull. 2004; 27: 38-46.

[11] IBM. IBM SPSS Statistics for Windows, Version 27. Armonk, NY: IBM Corp. 2020.

[12] Petrie A, Sabin C. Medical Statistics at a Glance (3rd edition, Vol. 23). Wiley-Blackwell. 2009.

[13] B Brauner, P Schwarz, M Wirth, F Gabor. Int. J. Pharm. 579 :2020;119158.

[14] M Samy, MG Ibrahim MG, Alalm M, Fujii Sep Purif. Technol. 249: 2020;117173.

[15] N Kraupner, S Ebmeyer, M Hutinel, J Fick, CF Flach, DGJ Larsson. Environ. Int. 2020; 144: 106083.

[16] Anatoly V. Skalny Lothar Rink Olga P. Ajsuvakova Michael Aschner Viktor A. Gritsenko Svetlana I. Alekseenko Andrey A. Svistunov Demetrios Petrakis Demetrios A. Spandidos Jan Aaseth Aristidis Tsatsakis Alexey A. Tinkov. Zinc and respiratory tract infections: Perspectives for COVID-19. International Journal of Molecular Medicine. 2020; 46: 17-26.

[17] Angelo Frei, Johannes Zuegg, Alysha G. Elliott, Et al. Metal complexes as a promising source for new antibiotics. Chem. Sci. 2020; 11: 262. 